\title{
Microbial diversity analysis of jiaoke from Xilingol, Inner Mongolia
}

\author{
Hui Fan, ${ }^{1,2}$ Rui Huo, ${ }^{1,2}$ Jingna Zhao, ${ }^{1,2}$ Tingting Zhou, ${ }^{1,2}$ Musu Zha, ${ }^{1,2}$ Lai-Yu Kwok, ${ }^{1,2}$ Heping Zhang, ${ }^{1,2}$ \\ and Yongfu Chen ${ }^{1,2 *}$ \\ ${ }^{1}$ Key Laboratory of Dairy Biotechnology and Engineering, Ministry of Education, Inner Mongolia Agricultural University, Huhhot 010018, China \\ ${ }^{2}$ Inner Mongolia Key Laboratory of Dairy Biotechnology and Engineering, Inner Mongolia Agricultural University, Hohhot 010018, China
}

\section{ABSTRACT}

Jiaoke is a traditional Mongolian fermented dairy product that is nutritious and has a unique taste. It is made from the fat separated from fermented milk. In this study, we collected 24 jiaoke samples from the Xilingol region of Inner Mongolia. The microbiota composition of the collected samples was analyzed using 16S rRNA small-molecule real-time sequencing, and the lactic acid bacteria (LAB) population was enumerated and isolated by laboratory culture techniques. We used an electronic tongue device to assess the taste quality of the products. One hundred fifty LAB isolates (5 genera and 14 species) were recovered and identified by $16 \mathrm{~S}$ rRNA sequencing across all samples. Lactococcus lactis and Lactobacillus plantarum accounted for $51.33 \%$ and $10.67 \%$ of the total isolates, respectively. The smallmolecule real-time sequencing of full-length $16 \mathrm{~S}$ rRNAs revealed an overall bacterial microbiota composition of 10 phyla, 121 genera, and 186 species, largely represented by sequences of Lactococcus $(68.46 \%)$ and Lactococcus lactis $(52.92 \%)$ at the genus and species levels, respectively. The electronic tongue analysis revealed that the sweetness, bitterness, sourness, and saltiness of jiaoke varied greatly between samples. The presence of Lactococcus lactis correlated positively with bitter aftertaste; the presence of Lactococcus piscium correlated positively with umami and negatively with astringent and bitter aftertastes; and the presence of Lactobacillus helveticus correlated positively with sourness and negatively with other taste qualities. These results suggest that the microbiota composition and product taste are closely related. The novel LAB strains collected in this work represent valuable natural microbial resources.

Key words: jiaoke, lactic acid bacteria, Mongolian, traditional fermented food, small-molecule real-time sequencing

Received September 12, 2019.

Accepted March 3, 2020.

*Corresponding author: nmgyfchen@126.com

\section{INTRODUCTION}

Mongolian traditional fermented dairy products, such as jiaoke, airag, koumiss, and vrum, have a long history. They are made of milk from domesticated animals, including cows, horses, goats, and sheep by traditional processing methods and natural microbial inocula. Jiaoke is a popular and unique traditional fermented dairy food handmade by herders. It is mostly available in regions where Mongolians live. The main composition of jiaoke comprises fat, moisture, and nonfat milk solids such as protein and trace elements (Zhu et al., 2017). Generally, fresh filtered milk is poured into a clean container to allow natural fermentation at room temperature. The container is stored in a cool place in summer and in a warm place in winter. After 1 to $2 \mathrm{~d}$, the oil will float to the top and form a cream on the milk surface. Then, the cream is gently collected and placed into a hanging white cotton bag to drain the whey. Fermentation of the cream continues for another 1 to $2 \mathrm{~d}$ with occasional stirring at ambient temperature (Gong et al., 2010). The appearance of typical jiaoke is shown in Figure 1. The final jiaoke product has a unique taste, extended shelf life, enriched nutrition, and contains a variety of beneficial substances (Yamei et al., 2019).

Jiaoke fermentation is a result of the metabolic action of natural lactic acid bacteria (LAB) from the raw milk, the container, and the direct environment (Gupta and Bajaj, 2017). The LAB population, which includes Lactobacillus (Lb.), Lactococcus (Lc.), Streptococcus (Strep.), and Leuconostoc (Leu.) play important roles in influencing the flavor and ripening process of fermented milk products (Casalta et al., 2009; Rein, 2018). For example, Dan et al. (2019) found that the Lactobacillus plantarum P-8 inhibits the generation of heptanal and promotes the formation of hexanoic acid (Dan et al., 2019). Wang et al. (2018) found that Lactobacillus and Prevotella contribute to the flavor formation of zhaguangjiao (a characteristic traditional fermented food of the ethnic minority in southwest China). Microorganisms in food also influence the biochemical profiles and taste quality of fermented products. For instance, 


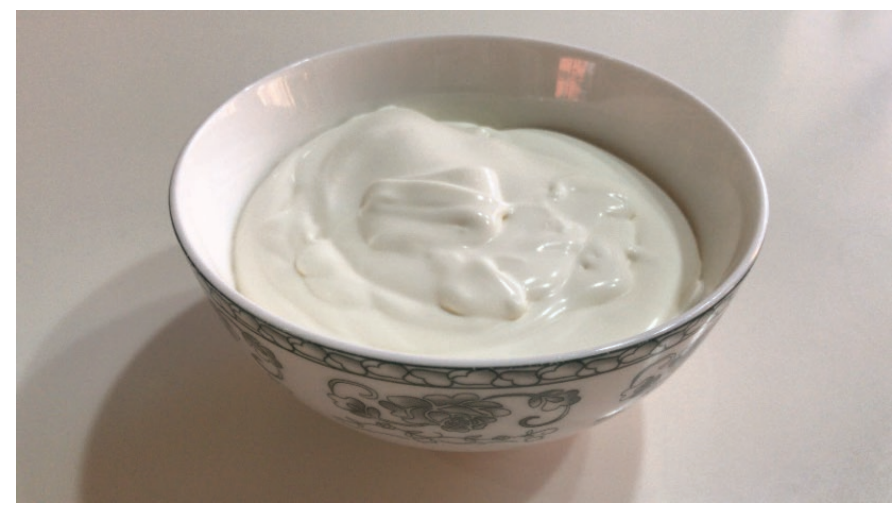

Figure 1. Appearance of typical jiaoke.

a metabolic fingerprinting study of cheese found that some amino acids and volatile metabolites are associated with the metabolism of Lc. lactis (Le Boucher et al., 2013), and the taste change during cheese ripening may be affected by bacterial metabolites, such as amino acids and fats (McSweeney, 2004; Piombino et al., 2008).

This study aimed to isolate and identify novel LAB by traditional laboratory culture methods, as well as to describe the bacterial microbiota of jiaoke based on the full-length 16S rRNA profile using single-molecule real-time (SMRT) sequencing technology. The taste of jiaoke was also determined by using an electronic tongue device. The overall objective was to identify correlations between the bacterial composition and the taste of jiaoke.

\section{MATERIALS AND METHODS}

\section{Sample Collection}

Twenty-four artisanal jiaoke samples were collected from 24 Mongolian households in the rural area of the Xilingol region of Inner Mongolia. Sample collection information is shown in Supplemental Table S1 (https://doi.org/10.3168/jds.2019-17583). For each jiaoke sample, 2 aliquots were collected. About $1.5 \mathrm{~mL}$ of jiaoke was put into a $2.5-\mathrm{mL}$ sterile cryovial that contained $0.5 \mathrm{~g}$ of sterile neutralizer $\left(\right.$ starch $/ \mathrm{CaCO}_{3}$, 50:1, wt/wt). The sample and neutralizer were mixed thoroughly and reserved for LAB isolation. A second aliquot of about $20 \mathrm{~mL}$ of jiaoke was placed in a sterile tube for other studies. The samples were transported to the laboratory in a cooler box by air. The $1.5-\mathrm{mL}$ samples reserved for enumeration and isolation of $\mathrm{LAB}$ were processed immediately, and the $20-\mathrm{mL}$ samples were stored at $-80^{\circ} \mathrm{C}$ until further analysis.

\section{Enumeration and Isolation of LAB}

The sample was diluted serially with saline $(0.85 \%$ $\mathrm{NaCl}$, wt/vol) to reach final concentrations of $10^{-4}$, $10^{-5}$, and $10^{-6}$. One milliliter of each diluted sample was plated on de Man, Rogosa, and Sharpe selective agar (Oxoid, Shanghai Harmony Biotechnology Co. Ltd., Shanghai, China) for Lactobacillus enumeration and isolation. Similarly, $0.2 \mathrm{~mL}$ of each diluted sample was spread on M17 agar for enumeration and isolation of lactococci (Oxoid, Shanghai Harmony Biotechnology Co. Ltd.). Cycloheximide and polymyxin B sulfate mixture at a final concentration of $10 \mu \mathrm{g} / \mathrm{mL}$ was added into agar plate to prevent fungal growth. Plates were incubated anaerobically for $48 \mathrm{~h}$ at $37^{\circ} \mathrm{C}\left(\mathrm{CO}_{2}: \mathrm{H}_{2}\right.$ : $\left.\mathrm{N}_{2}=10: 10: 80\right)$. After incubation, plates with colonies ranging between 30 and $300 \mathrm{cfu} / \mathrm{mL}$ were counted and recorded. Colonies with distinct morphologies were selected and purified by streaking on a new agar plate of the same culture medium. Purified gram-positive, catalase-negative bacterial isolates were preserved in skim milk (10\%, wt/vol) containing $0.1 \%$ (wt/vol) yeast extract as a cryoprotectant and stored at $-80^{\circ} \mathrm{C}$.

\section{Identification of Isolates by 16S rRNA Gene Sequencing}

Total genomic DNA of each bacterial isolate was extracted by using the TIANamp Bacteria DNA kit (Tiangen Biotech Co. Ltd., Beijing, China). The concentration and quality of extracted DNA were determined by spectrophotometry. The $16 \mathrm{~S}$ rRNA gene of each isolate was amplified by PCR using the primers $27 \mathrm{~F}$ (5'-GCAGAGTTCTCGGAGTCACGAAGAGTTTGATCCTGGCTCAG-3') and 1495R (5'-AGCGGATCACTTCACACAGGACTACGGCTACCTTGTTACGA-3') (Mosher et al., 2013). The 50- $\mu \mathrm{L}$ reaction system contained $1.5 \mu \mathrm{L}$ of template DNA, $5 \mu \mathrm{L}$ of $10 \times$ PCR buffer with $4 \mu \mathrm{L}$ of dNTPs $(2.5 \mathrm{mmol} / \mathrm{L}), 1.5 \mu \mathrm{L}$ of $1495 \mathrm{R}(10 \mathrm{mmol} / \mathrm{L}), 1.5 \mu \mathrm{L}$ of $27 \mathrm{~F}(10 \mathrm{mmol} / \mathrm{L})$, $0.5 \mu \mathrm{L}$ of Taq DNA polymerase $(5 \mathrm{U} / \mu \mathrm{L})$, and 36 $\mu \mathrm{L}$ of sterilized ultra-pure water. Thermocycling was performed on an automatic thermal cycler (PTC-200, MJ Research, Waltham, MA) with the following conditions: predenaturation at $94^{\circ} \mathrm{C}$ for $5 \mathrm{~min} ; 30$ cycles of denaturation at $94^{\circ} \mathrm{C}$ for $1 \mathrm{~min}$, annealing at $58^{\circ} \mathrm{C}$ for $1 \mathrm{~min}$, and extension at $72^{\circ} \mathrm{C}$ for $2 \mathrm{~min}$; followed by an extension step at $72^{\circ} \mathrm{C}$ for $10 \mathrm{~min}$ (Liu et al., 2012). The amplicons were sequenced by Majorbio Bio-Pharm Technology Corporation Ltd. (Shanghai, China). The $16 \mathrm{~S}$ sequence analysis was performed with the SeqMan software and searched across the National Center for Biotechnology Information (NCBI) database using the 
BLAST program (http://www.ncbi.nlm.nih.gov/) to identify the isolate. A phylogenetic tree was built based on sequences generated in this work and downloaded sequences of reference strains, including Lc. lactis ssp. lactis ATCC $19435^{\mathrm{T}}$, Strep. thermophilus ATCC $19258^{\mathrm{T}}$, Enterococcus faecalis ATCC $19433^{\mathrm{T}}$, Lb. helveticus ATCC $15009^{\mathrm{T}}$, Lb. casei ssp. casei ATCC $393^{\mathrm{T}}$, and Lb. plantarum ATCC $14917^{\mathrm{T}}$ ) from the Mega software version 7.0 (Kumar et al., 2016).

\section{Determination of $\mathrm{pH}$, Titratable Acid Content, and Taste of Jiaoke}

The $\mathrm{pH}$ of the samples was measured using a PHS-3E pH meter (Leici, Shanghai, China). The level of titratable acid was determined according to national food safety standards in China (National Standards of the People's Republic of China, 1996).

An electronic tongue (SA-402B, Intelligent Sensor Technology Co. Ltd., Atsugi, Japan) was used to measure 9 taste parameters of jiaoke: sourness, saltiness, bitterness, umami, astringent, sweetness, aftertaste-A (astringent), aftertaste-B (bitterness), and richness, respectively (Wang et al., 2018). Briefly, $5 \mathrm{~g}$ of each collected sample was added to $195 \mathrm{~mL}$ of distilled water with stirring, and the mixture was boiled for $5 \mathrm{~min}$. The mixture was then stored at $4^{\circ} \mathrm{C}$ for $24 \mathrm{~h}$ and the supernatant was collected and filtered. The electronic tongue was activated $24 \mathrm{~h}$ before being used for measurement. The baseline level was set using a reference sample. Then, each jiaoke sample was poured into a sample cup for measurement. The taste values of jiaoke were calculated as the difference between the sample readout and the baseline value.

\section{SMRT Sequencing}

Cluster analysis was performed based on the measured taste parameters partitioning around the group clustering algorithm. The optimal number of clusters was determined using the Caliński-Harabasz index (Caliński and Harabasz, 1974). Then, 14 samples were selected for SMRT sequencing from the 3 clusters based on sample proximity to the cluster center. The 14 samples included XM9, XM10, XM11, and XM18 (from cluster 1); XM2, XM5, XM6, XM12, XM15, and XM19 (from cluster 2); and XM23, XM25, XM26, and XM29 (from cluster 3). The microbial genomic DNA of jiaoke was extracted by using the Omega DNA isolation kit (Omega Bio-Tek, Norcross, GA). The DNA quality was checked by $1 \%$ agarose gel electrophoresis and spectrophotometer (Thermo Fisher Scientific, Waltham, MA; Hui et al., 2017). The final DNA concentration was $>100 \mathrm{ng} / \mu \mathrm{L}$, and the optical density
(OD) ratio at 260 and $280 \mathrm{~nm}$ was between 1.8 and 2.0. The full-length region of the $16 \mathrm{~S}$ rRNA gene of the sample was amplified via PCR using a forward primer 27F (5'-GAGAGTTTGATCCTGGCTCAG-3') and a reverse primer 1541R (5'-AAGGAGGTGATCCAGCCGCA-3') (Adelowo and Fagade, 2009) that contained 16-bp barcodes to distinguish between samples. The PCR programs were as follows: predenaturation at $95^{\circ} \mathrm{C}$ for $4 \mathrm{~min}$; denaturation at $95^{\circ} \mathrm{C}$ for $1 \mathrm{~min}$, annealing at $60^{\circ} \mathrm{C}$ for $45 \mathrm{~s}$, extension at $72^{\circ} \mathrm{C}$ for $1 \mathrm{~min}$; cycles were repeated 30 times, followed by an extension step at $72^{\circ} \mathrm{C}$ for $7 \mathrm{~min}$ (Quigley et al., 2012). These amplicons were used to construct DNA libraries by using the Pacific Biosciences (Menlo Park, CA) SMRT bell template prep kit 1.0 (Mosher et al., 2013). Sequencing was performed using $\mathrm{P} 6$-C4 chemistry on a PacBio RS II instrument (Pacific Biosciences) in accordance with the manufacturer's instructions.

\section{Bioinformatics and Statistical Analyses}

The raw data were processed using the RS-ReadsOfinsert.1 procedure in the SMRT Portal (version 2.7, Pacific Biosciences). The sequence filter parameters were based on a previous study (Hou et al., 2015). Species and diversity analyses were performed on the acquired high-quality sequences by the Quantitative Insights Into Microbial Ecology (QIIME) package (version 1.7; Caporaso et al., 2010b). The high-quality sequence alignment was performed using PyNAST (Caporaso et al., 2010a) and UCLUST (Edgar, 2010) software according to $100 \%$ clustering of sequence identity. Sequences were classified into operational taxonomic units (OTU) under a threshold of 97\% identity. The Ribosomal Database Project (RDP, release 11.5; Cole et al., 2007) and Greengenes (version 13.8; DeSantis et al., 2006) databases were applied to determine the phylogeny of the OTU at a minimum bootstrap threshold of $80 \%$ (Hou et al., 2015). The UniFrac distance was calculated based on the phylogenetic tree (Lozupone and Knight, 2005). Principal coordinate analysis (PCoA) was performed based on both weighted and unweighted UniFrac distances. Analysis of $\alpha$ - and $\beta$-diversity was performed based on the de novo taxonomic tree constructed by the representative chimera-checked OTU set using FastTree (Price et al., 2009). Shannon-Wiener, Chao1, Simpson, and rarefaction estimators were calculated to evaluate sequencing depth and microbial diversity (Hou et al., 2019).

Statistical analyses were performed using $\mathrm{R}$ packages (http://www.rproject.org/) and Matlab (v2011b; MathWorks Inc., Natick, MA). Graphics were generated using the R "ggplot2" and "corrplot" packages (Wickham, 2016) and the Origin software (version 7.00; 
OriginLab Corp., Hampton, MA). Differences in taste indices between groups were evaluated using MannWhitney test, and the diversity indices were evaluated by Kruskal-Wallis test. Pearson correlation analysis was performed between the jiaoke bacterial microbiota and taste parameters.

\section{Nucleotide Sequence Accession Numbers}

All of the SMRT sequencing data reported in this study were deposited to the MG-RAST database (project number: mgp92388, http://metagenomics.anl.gov/ ). Sequences of individual LAB isolates were submitted to the NCBI database under accession numbers MF893765 to MF893894.

\section{RESULTS}

\section{Taste Quality Detected by Electronic Tongue}

Nine taste quality parameters of jiaoke - sourness, bitterness, saltiness, umami, astringency, sweetness, aftertaste-A, aftertaste-B, and richness - were measured and normalized against values determined by a reference electrode. Four of the tastes were substantially different between samples: sweetness (ranging from -17.15 to 0.00 ), sourness (ranging from -3.59 to 6.01 ), bitterness (ranging from -5.33 to 1.72), and saltiness (ranging from -1.17 to 5.42) (Figure 2). Cluster analysis of jiaoke taste revealed that the 24 samples could be divided into 3 clusters.

The Mann-Whitney test was used to analyze differences in the taste indicators among the 3 clusters. Cluster 1 had a significantly higher degree of saltiness, sweetness $(P<0.01)$, bitterness, umami, and richness $(P<0.05)$ than clusters 2 or 3 , while the opposite trend was observed for sourness $(P<0.05)$. No significant difference was observed in astringency, aftertaste-A, or aftertaste-B among the 3 clusters.

\section{Acidity and LAB Counts of Jiaoke}

The viable counts of $\mathrm{LAB}$ ranged from $10^{4}$ to $10^{7}$ $\mathrm{cfu} / \mathrm{g}$ across samples. The average $\mathrm{pH}$ of the jiaoke samples was 3.89 , ranging from $3.54 \pm 0.00$ to $4.30 \pm$ 0.09 . The average titratable acidity was $124.00^{\circ} \mathrm{T}$.

\section{Isolation and Identification of LAB}

A total of 150 gram-positive and catalase-negative LAB were isolated, including 33 rod-shaped isolates and 117 coccal isolates. The $16 \mathrm{~S}$ rRNA genes of these isolates were sequenced, and their identities were deter- mined by BLAST search on NCBI, using a threshold of $>99 \%$ similarity to the type strains.

These 150 isolates were assigned to 14 species and subspecies (Table 1), including $77 \mathrm{Lc}$. lactis isolates (51.33\% of total isolates) and $16 \mathrm{Lb}$. plantarum isolates $(10.67 \%)$. Other species (number of isolates arranged in descending order) included $L b$. casei, Lb. helveticus, Strep. thermophilus, Enterococcus (Ent.) faecalis, Lb. garvieae, Ent. faecium, Ent. italicus, Leu. lactis, Lb. brevis, Ent. gallinarum, Ent. gilvus, and Strep. gallolyticus.

Then, phylogenetic analysis was performed on these isolates and on selected type strains. The selected isolates represented 6 different species (Lc. lactis ssp. lactis, Strep. thermophilus, Ent. faecalis, Lb. helveticus, Lb. casei ssp. casei, and Lb. plantarum), and corresponding reference strains were used to construct a phylogenetic tree (Figure 3).

\section{Jiaoke Microbiota}

A total of 57,004 high-quality $16 \mathrm{~S}$ rRNA sequences were obtained from 14 jiaoke samples (average of 4,071 sequences per sample; ranging from 1,796 to 7,406). At the OTU level, a total of 3,311 OTU were identified (average of 236 OTU per sample). The Chao1 index of the investigated jiaoke samples ranged from 176.35 to 640.74 (average of 379 sequences per sample; XM2 and XM29 had the highest and the lowest microbial richness, respectively). The Shannon and Simpson indices were used to analyze the microbial diversity of the samples. Sample XM2 had the highest microbial diversity (Shannon and Simpson indices were 4.65 and 0.82 , respectively), whereas sample XM19 had the lowest microbial diversity (Shannon and Simpson indices were 2.10 and 0.44 , respectively).

The rarefaction curves of the samples did not reach saturation, indicating that new bacterial species might be found by increasing the sequencing depth. However, the Shannon-Wiener diversity curves of all samples leveled off, suggesting that the sequence depth was adequate for capturing representative microbiota composition. Large variations existed in bacterial richness and evenness in jiaoke, as indicated by the rank-abundance curves.

Based on SMRT sequencing, the jiaoke microbiota comprised 10 bacterial phyla, 21 classes, 34 orders, 63 families, 121 genera, and 186 species. The relative abundances of major taxa detected in the microbiota are shown in Figure 4, which included taxa with a relative abundance $>1 \%$. The major phyla of the jiaoke microbiota were Firmicutes $(87.17 \%)$ and Proteobacteria (11.40\%). The 3 most prevalent genera were Lactococcus $(76.50 \%)$, Streptococcus (9.97\%), and Lactobacillus 
(a)

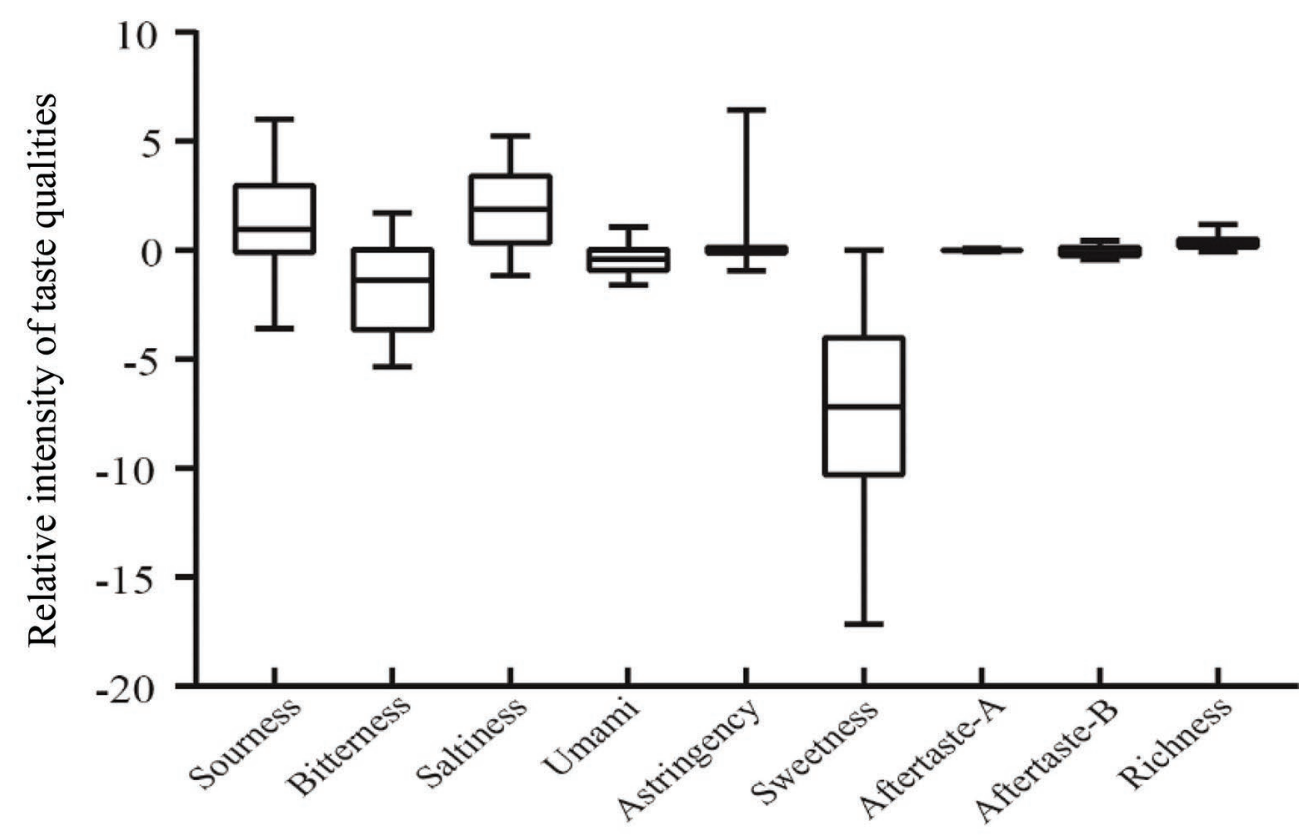

(b)

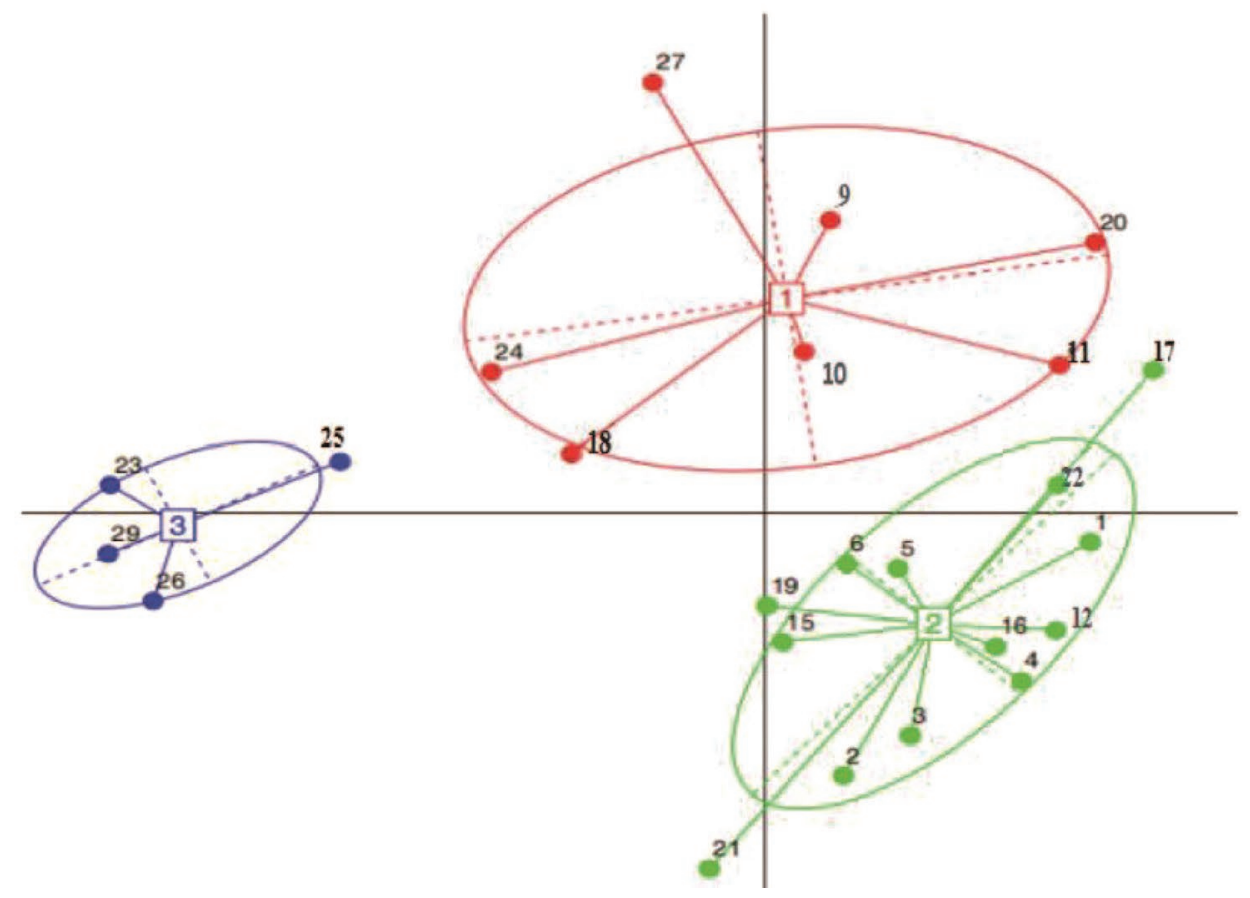

Figure 2. (a) Boxplots showing variations of 9 taste qualities of jiaoke; (b) cluster analysis of samples based on taste qualities. The box indicates the data from $25 \%$ to $75 \%$, the line indicates the median, and the whiskers indicate the minimum and maximum. The aftertaste-A is astringent of aftertaste, and aftertaste-B is bitterness of aftertaste. The numbers in squares represent clusters, and the other numbers represent the samples.

(2.39\%). Lactococcus lactis was the main bacterial species $(67.93 \%)$ detected in the jiaoke samples. Sequences representing 7 species were commonly shared across all samples, including Lc. lactis, Acinetobacter johnsonii, Klebsiella (K.) oxytoca, Lb. helveticus, Strep. parauberis, Leu. mesenteroides, and Enhydrobacter aerosaccus. Five core species (Lc. lactis, Lb. helveticus, Lc. raffinolactis,
Lc. piscium, and Lc. garvieae) were identified in the jiaoke microbiota.

A large proportion of the total sequences represented LAB (87.73\%), including 5 major genera; namely Lactococcus, Streptococcus, Lactobacillus, Enterococcus, and Leuconostoc. The most dominant genus was Lactococcus (39,790 high-quality sequences). Sequences 


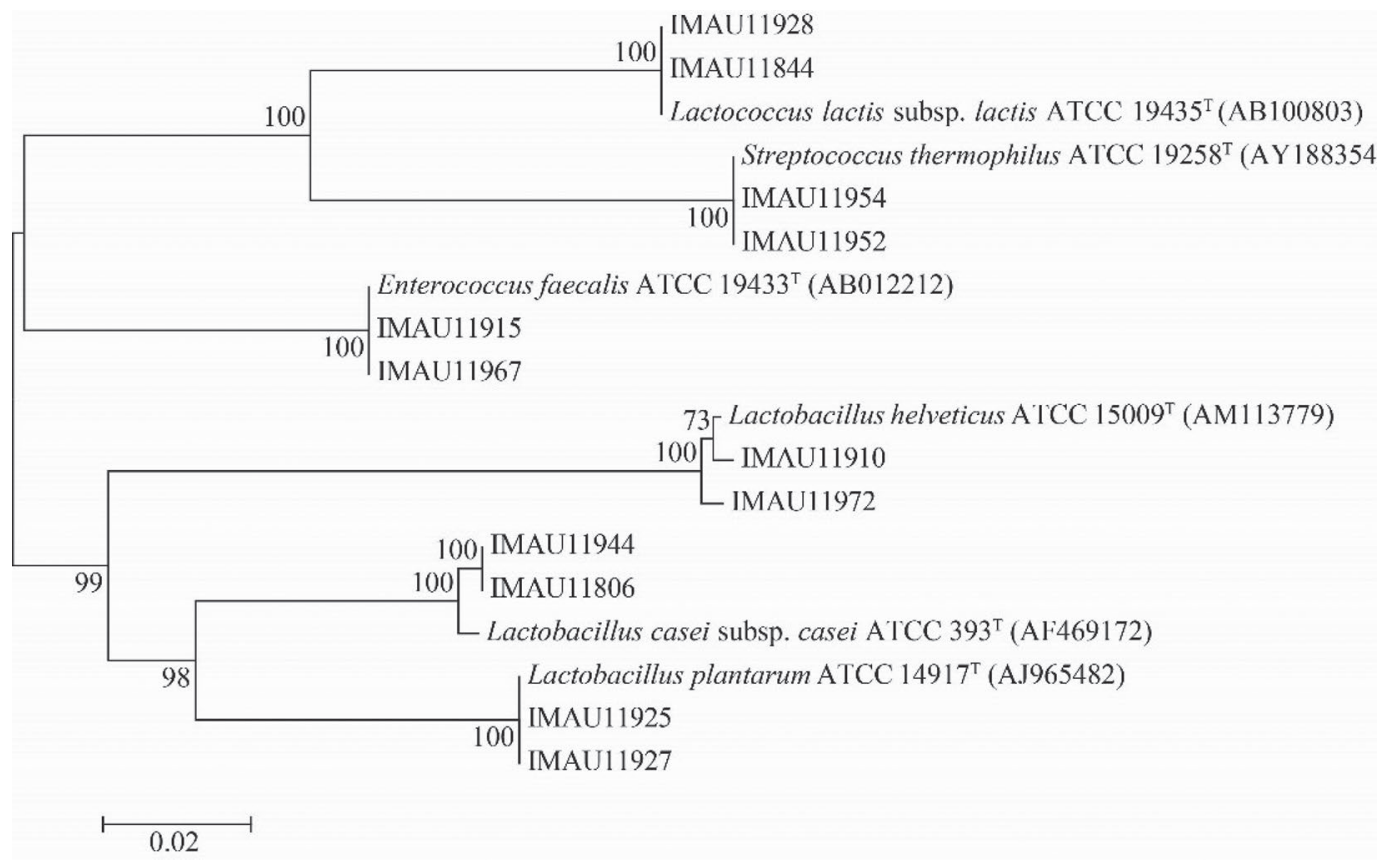

Figure 3. Phylogenetic analysis based on 16S rRNA genes of selected lactic acid bacteria isolated from jiaoke and type strains.

representing 9 Lactobacillus species, 9 Streptococcus species, 7 Enterococcus species, 5 Leuconostoc species, and 5 Lactococcus species were detected in the jiaoke microbiota. No sequences represented the genera Weissella and Pediococcus. Finally, low-abundant potential pathogen sequences were also detected, including Macrococcus caseolyticus, Acinetobacter guillouiae, Arcobacter butzleri, Enhydrobacter aerosaccus, and K. pneumoniae. Slightly different results were obtained by applying conventional laboratory culture methods. Lactobacillus helveticus and Lc. lactis were recovered in most samples; and Leu. lactis, Lb. brevis, and Ent.

Table 1. Taxonomic distribution of lactic acid bacteria isolated from jiaoke fermented dairy product

\begin{tabular}{lc}
\hline Species & No. of detected isolates \\
\hline Lactococcus lactis & 77 \\
Lactococcus garvieae & 7 \\
Lactobacillus casei & 8 \\
Lactobacillus helveticus & 8 \\
Lactobacillus plantarum & 16 \\
Lactobacillus brevis & 1 \\
Enterococcus faecalis & 10 \\
Enterococcus faecium & 5 \\
Enterococcus gallinarum & 1 \\
Enterococcus italicus & 3 \\
Enterococcus gilvus & 1 \\
Streptococcus thermophilus & 10 \\
Streptococcus gallolyticus & 1 \\
Leuconostoc lactis & 2 \\
Total & 150 \\
\hline
\end{tabular}

italicus were isolated only in some samples. A high abundance of Lactococcus was consistently detected by both SMRT sequencing and traditional LAB cultivation methods.

\section{Features of 3 Taste Clusters of Jiaoke Bacterial Microbiota}

Based on the taste profile, the jiaoke samples could be divided into 3 clusters. Generally, cluster 2 exhibited a higher Chao1 index and higher number of observed OTUs compared with the other 2 clusters (Figure 5a and $5 \mathrm{~b}$ ). By analyzing the unweighted UniFrac distance of the bacterial microbiota by PCoA, the samples in cluster 2 were mainly distributed in the left quadrants and those in clusters 1 and 3 were mainly distributed to the right side, suggesting an overall different microbiota in cluster 2 samples compared with other clusters (Figure 5c). This trend was not as obvious when the weighted UniFrac distance was analyzed (Figure 5d), suggesting that variation in microbiota composition was likely due to the abundant taxa present in jiaoke microbiota.

The microbiota of 3 jiaoke taste clusters were more similar at the phylum and genus levels (Figure 6a); greater variation was shown at the species level (Figure 6b). For example, cluster 2 was enriched with Lc. lactis and $L b$. helveticus, whereas clusters 1 and 3 had more Lc. lactis and Lc. piscium. Moreover, cluster 2 had the most Lc. raffinolactis, and cluster 1 had the least Strep. 
Phylum

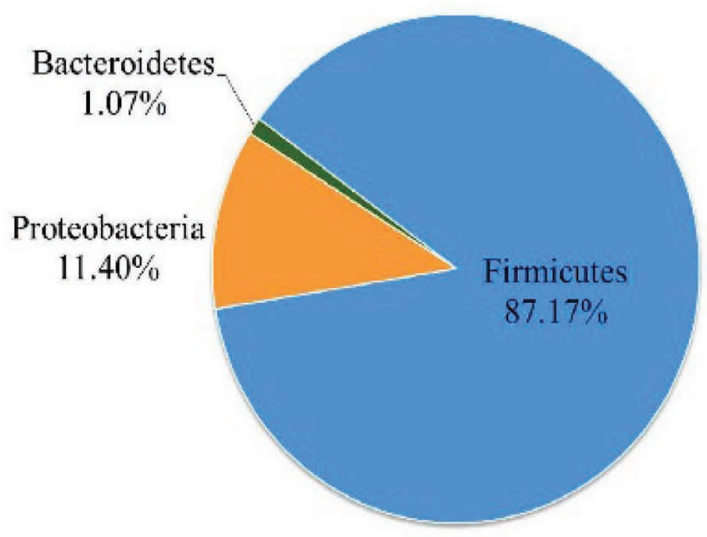

\section{Order}

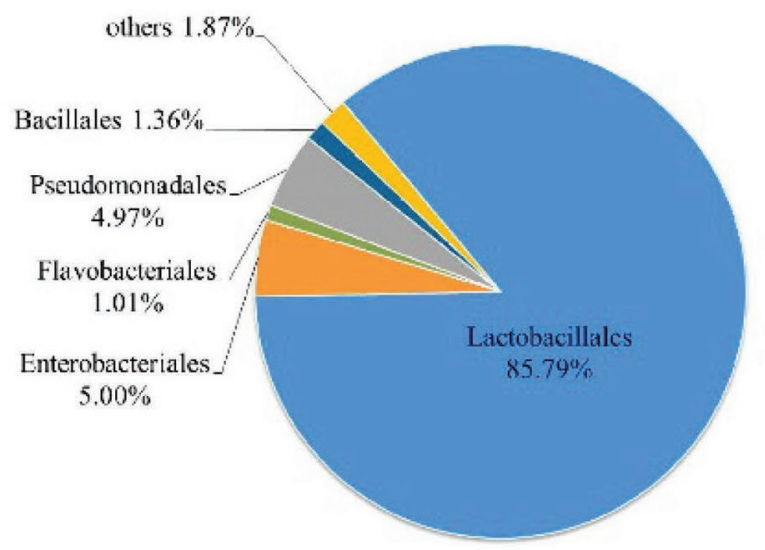

Family

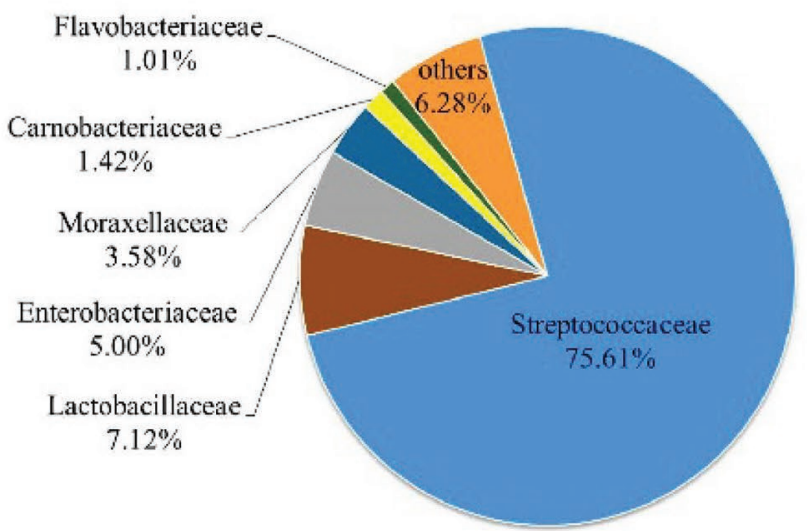

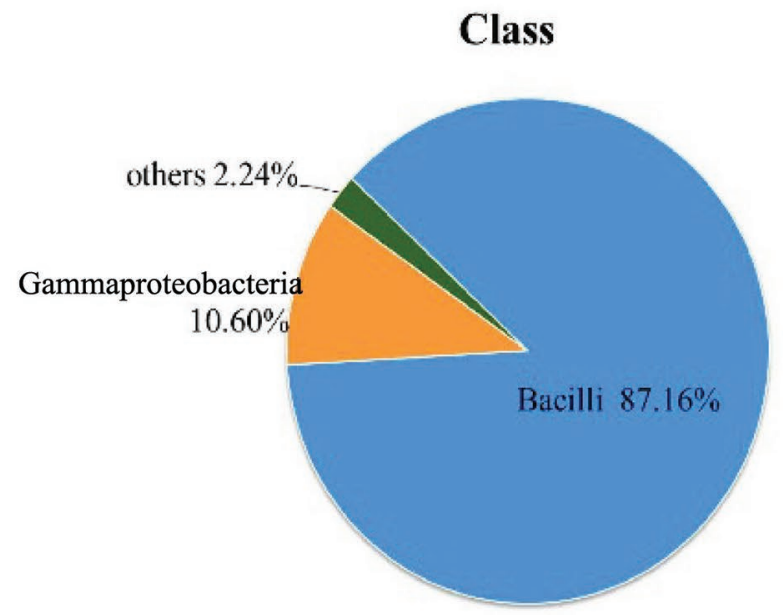

Genus
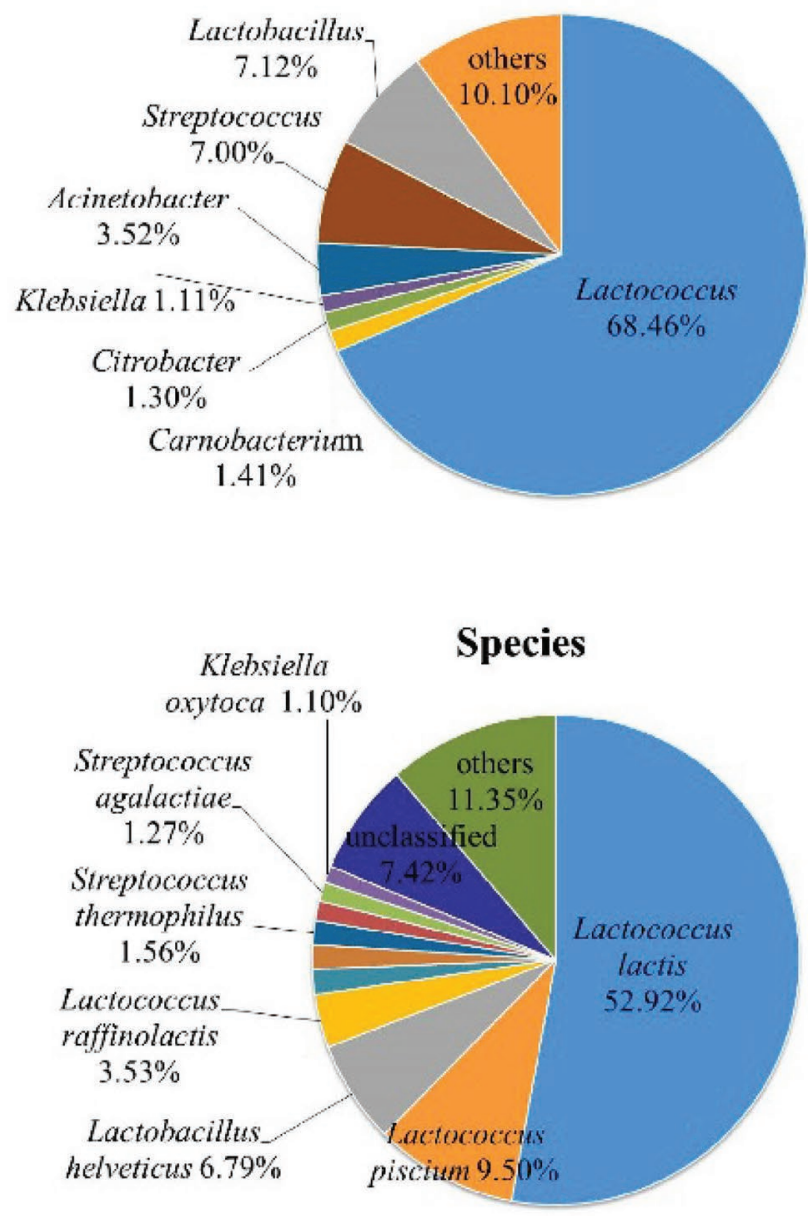

Figure 4. Bacterial composition of jiaoke microbiota at the phylum, class, order, family, genus, and species levels. 

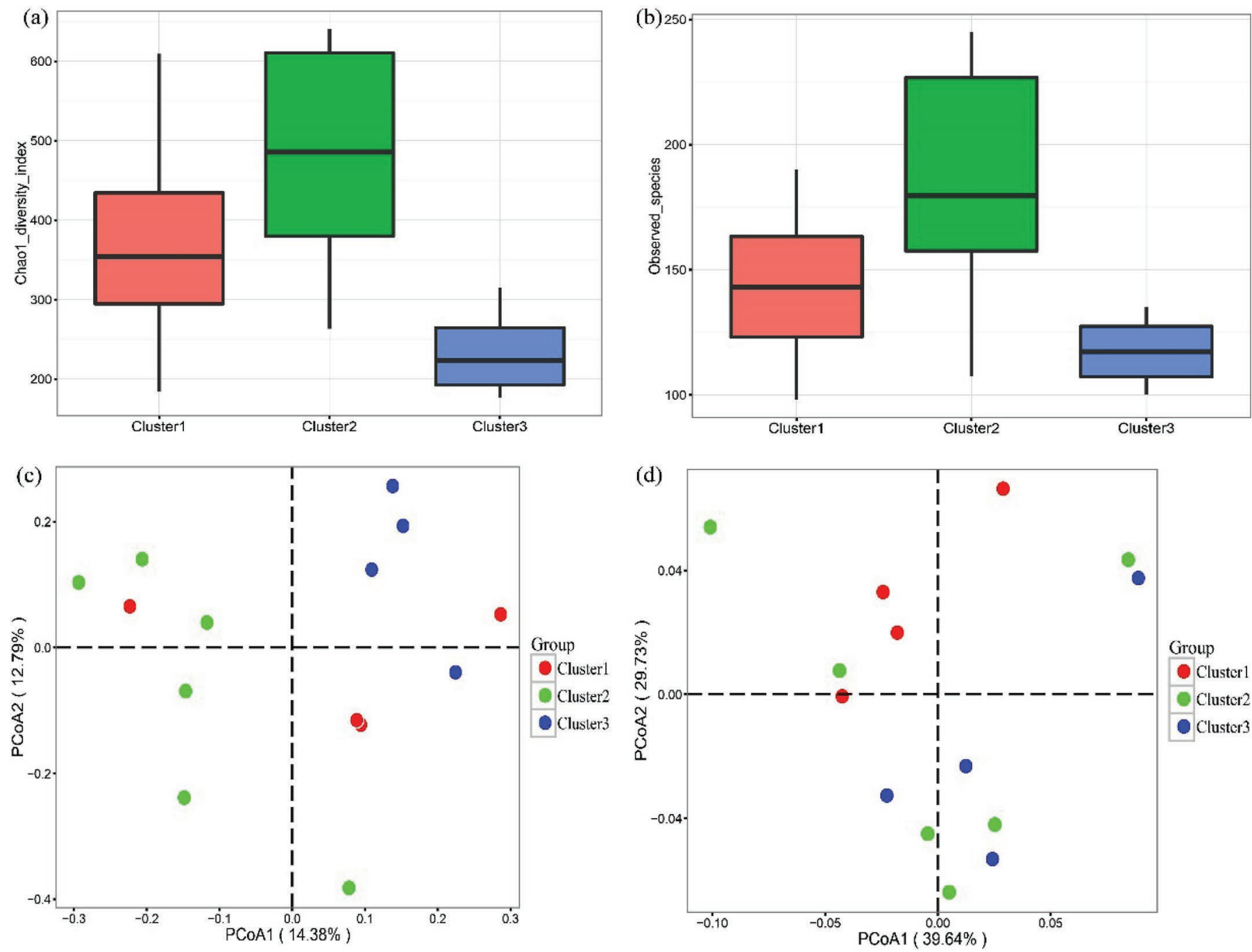

Figure 5. $\alpha$-Diversity indices of 3 clusters grouped by taste. (a) Chao 1; and (b) number of observed species; (c, d) principal coordinate analysis (PCoA) of jiaoke microbiota based on unweighted (c) and weighted (d) UniFrac distances. The box indicates the data from $25 \%$ to $75 \%$, the line indicates the median, and the whiskers indicate the minimum and maximum.

parauberis. Differences in the relative abundance of 10 species in the 3 taste clusters are shown in Figure 6.

\section{Correlation Between LAB and Jiaoke Taste Attributes}

To identify microbial species that potentially contributed to the taste of jiaoke, Spearman's correlation analysis was performed and the results are presented in a heatmap (Figure 7a). The correlation network between significant correlations and jiaoke taste is presented in Figure 7b. Positive correlations were found between several microbial sequences and taste attributes: Ent. camelliae with sourness and aftertaste-A $(P<0.05$, r $>0.5)$; Lc. lactis with aftertaste-B $(P<0.05, \mathrm{r}>0.5)$; and Lc. piscium and Leu. mesenteroides with umami $(P$
$<0.05, \mathrm{r}>0.5)$. Negative correlations were found for Lc. piscium with aftertaste-A and for Strep. agalactiae with richness, bitterness, and aftertaste-B $(P<0.05, \mathrm{r}$ $>0.5$ in all cases). Lactobacillus helveticus was negatively correlated with bitterness, saltiness, and umami $(P<0.05, \mathrm{r}>0.5)$. A positive correlation was found between Lc. piscium, Leu. mesenteroides, and umami $(P<0.05, \mathrm{r}>0.5)$.

\section{DISCUSSION}

Microorganisms play an active role in food fermentation by metabolizing raw food components and contributing to the taste of fermented products. Thus, it is of interest to study the microbiota composition of fermented food. Jiaoke is a traditional handmade 
fermented food from Mongolian. The major phyla of jiaoke microbiota were Firmicutes, Proteobacteria, and Bacteroidetes; this composition is very similar to that of other fermented dairy products such as koumiss $(\mathrm{Wu}$ et al., 2009) and Buryatian cheese (Jin et al., 2018). At the lower taxonomic levels, we found that a large proportion of the jiaoke microbiota was made up of LAB (83.73\% of the total sequences). The results obtained by SMRT sequencing and conventional culture methods were largely consistent.

Most commercial yogurt products in China contain a high amount of viable LAB (ranging from $10^{6}$ to $10^{9}$ $\mathrm{cfu} / \mathrm{mL}$; Ma et al., 2009). The viable counts of LAB in most investigated jiaoke samples were also within this range $\left(>10^{6} \mathrm{cfu} / \mathrm{g}\right)$, suggesting that these products could serve as a rich source of natural LAB. Some variations were found in viable counts of $\mathrm{LAB}, \mathrm{pH}$, and titratable acidity among samples, which may result from differences in heating process, raw milk source, and the addition of other ingredients when the products were made.

Some reports have analyzed the microbial diversity of fermented dairy products of Inner Mongolia. For example, Wu et al. (2009) reported that the dominant lactobacilli species in koumiss of Inner Mongolia were Lb. casei, Lb. helveticus, and Lb. plantarum. Liu et al. (2012) studied tarag samples in the eastern part of Inner Mongolia and found that Lb. helveticus, Lc. lactis ssp. lactis, and $L b$. casei were the most dominant LAB in these samples. Very few works have studied the microbial composition of jiaoke. Here, our data showed that jiaoke had a similar LAB composition to other traditionally fermented dairy products from Inner Mongolia, include 5 genera (Lactococcus, Lactobacillus, Enterococcus, Streptococcus, and Leuconostoc) and 14 species, among which $L c$. lactis and $L b$. plantarum were the most dominant. The rich LAB diversity and richness suggest that they are valuable resources for LAB isolation and selection for further identification of probiotics. However, Lc. lactis ssp. lactis was reportedly the predominant LAB in initial stages of Kurdish cheese ripening, whereas Ent. faecium and Lb. plantarum predominated in the later stages (Milani et al., 2017). Gesudu et al. (2016) attributed the difference in the bacterial structure of 22 koumiss samples to the geographical location of the sampling area. Similarly, the geographic site of samples affected microbiota composition in traditional fermented vegetables (Nam et al., 2012) and fermented milk (Watanabe et al., 2008; Sun et al., 2014). Li et al. (2017) found that cheeses from Kazakhstan, Belgium, Russia, and Italy had unique microflora compositions, which might be related to the cheese origin, production process, milk source, or fermentation environment. The manufacturing process and ripening conditions affected the microbial taxonomy of Austrian artisanal hard cheese (Schornsteiner et al., 2014). These factors might also be causing differences in the microbiota composition between jiaoke and other traditionally fermented dairy products, as well as jiaoke samples collected from different districts.

The SMRT sequencing identified 10 phyla, 121 genera, and 186 species in the bacterial microbiota of jia-
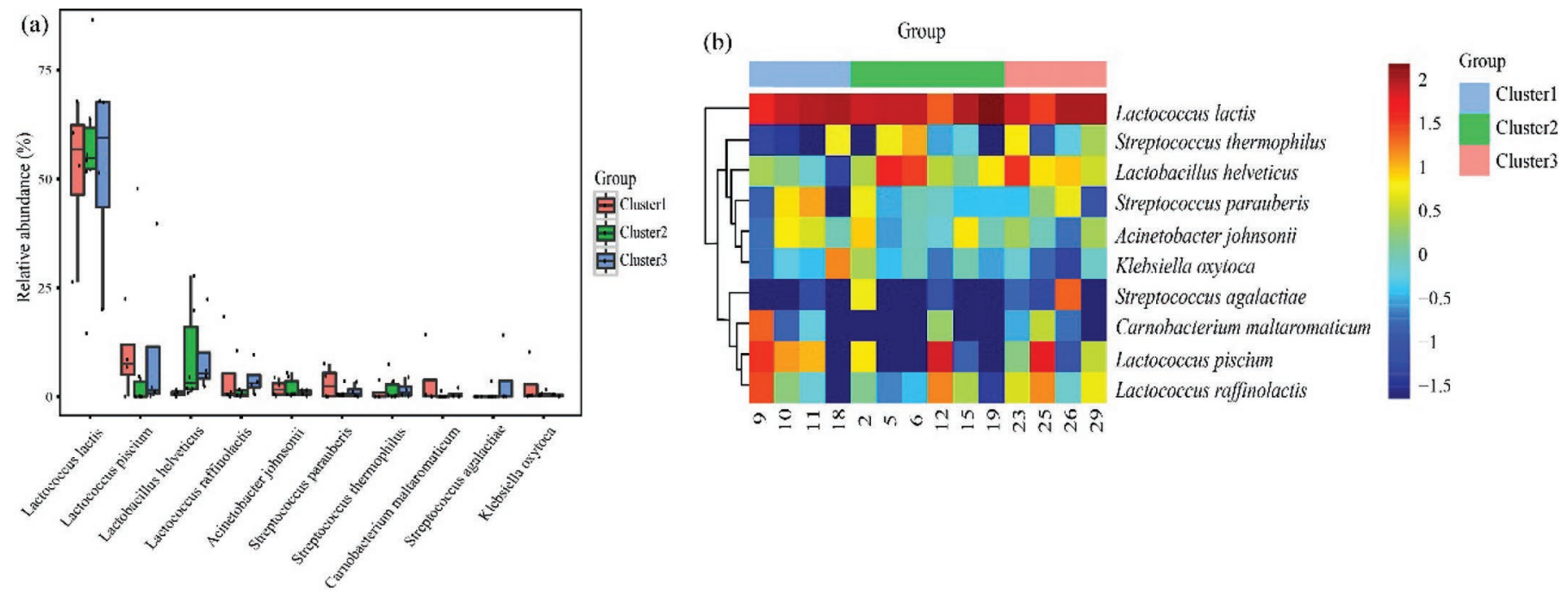

Figure 6. Comparison of jiaoke microbiota composition of 3 taste clusters at the species level. (a) Boxplots; and (b) heatmap showing relative abundances of 10 major species; each species comprised $>1 \%$ of total sequences. The box represents the data from $25 \%$ to $75 \%$, the line represents the median, the whiskers represent the minimum and maximum, the points represent samples, and the points outside the box represent outliers of samples. 

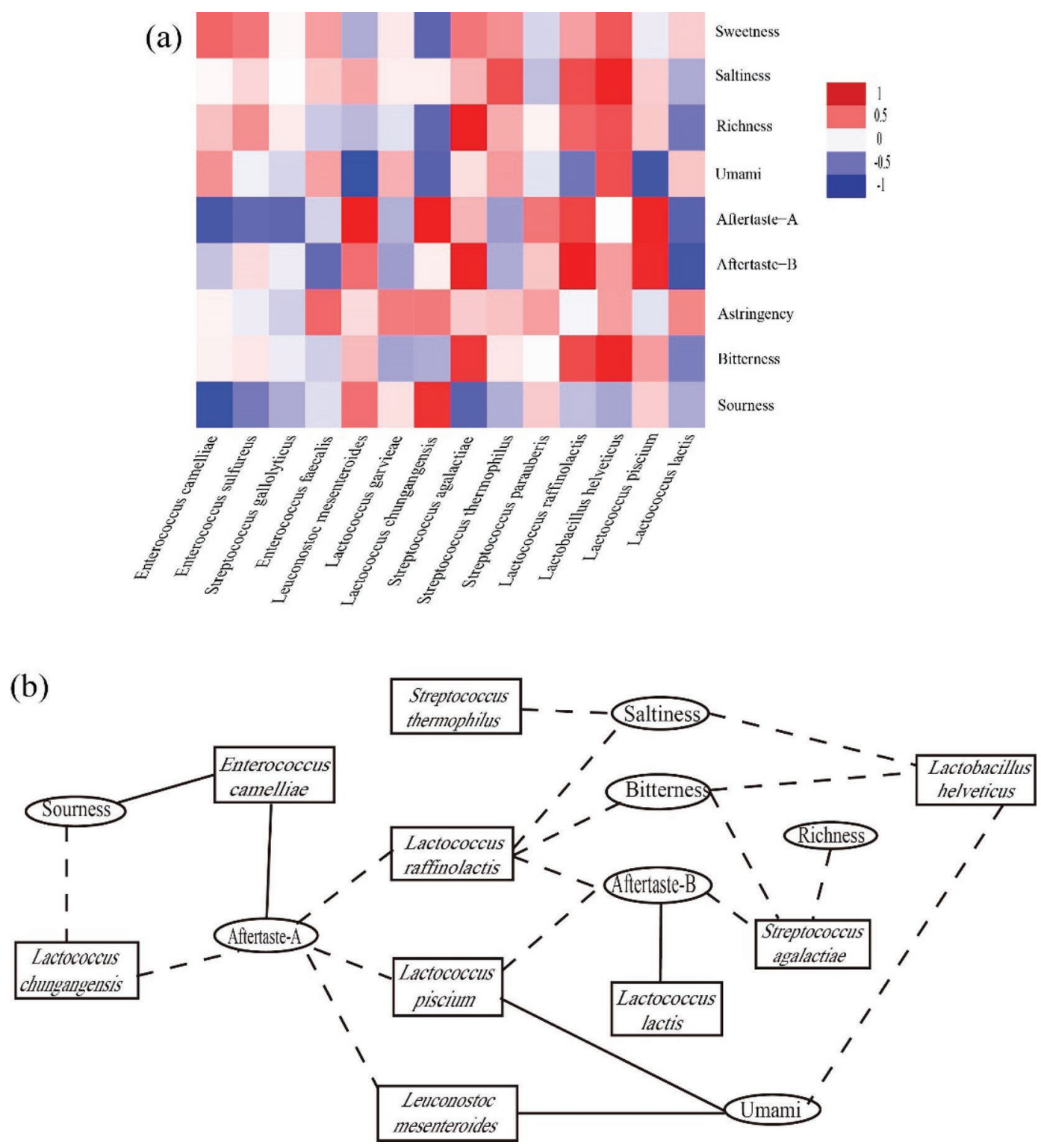

Figure 7. (a) Heatmap showing correlations between jiaoke microbial sequences and different taste quality; the analysis only included species of relative abundance $>0.01 \%$. (b) Network diagram showing correlations between identified jiaoke lactic acid bacteria sequences and taste attributes; solid and dotted lines indicate positive $(P<0.05, \mathrm{r}>0.5)$ and negative $(P<0.05, \mathrm{r}<-0.5)$ correlations, respectively.

oke. The number of sequences per sample was between 1,796 and 7,406 . The sequencing depth met the analytical requirements, although an increase in sequencing depth would probably help detect more low-abundant taxa. The abundance of bacterial sequences detected in the jiaoke microbiota seemed to be slightly lower than the number in Kazakh cheese ( $\mathrm{Li}$ et al., 2017), and higher than that in koumiss (Gesudu et al., 2016). This might be because some bacteria cannot easily metabolize the high fat in jiaoke or grow under the highly acidic environment of sour milk. The dominant bacterial sequences of the jiaoke were Firmicutes (87.17\%),
Proteobacteria (11.40\%), and Bacteroidetes (1.07\%), which were consistent with some dairy products such as Poro cheese and naturally fermented cow milk (AldreteTapia et al., 2014; Liu et al., 2015). The dominant genera were Lactococcus (68.46\%), Lactobacillus (7.12\%), and Streptococcus (7.00\%); and the dominant species were Lc. lactis $(52.92 \%)$, Lc. piscium $(9.50 \%)$, and $L b$. helveticus $(6.79 \%)$. The dominant species, Lactococcus lactis, is commonly used as a dairy starter because of the rapid acidification and curdling properties. This species also facilitates degradation of casein to produce aroma and thus improves the sensory quality of dairy 
products (Cavanagh et al., 2015; del Rio et al., 2015). The SMRT sequencing results were largely consistent with those obtained by culture methods. The SMRT sequencing is advantageous in describing the overall microbiota profile because some microbes might not be cultivable in the laboratory, whereas culture methods reflect the viability of detected bacteria. Nevertheless, each approach is limited by intrinsic bias caused by, for example, bacterial cultivation or sequencing. Moreover, viable-but-nonculturable cells might be generated during the fermentation process and they are often underdetected. The detection of cells of this status would require methods other than traditional culture-based methods and conventional $16 \mathrm{~S}$ rRNA sequencing; thus, this spectrum of cells was not addressed in the current work (Quirós et al., 2009).

The taste of jiaoke was analyzed by electronic tongue, and samples could be divided into 3 taste clusters. Cluster 1 was enriched with Lc. lactis, Lc. piscium, Lc. raffinolactis, and Strep. parauberis; cluster 2 was enriched with Lc. lactis, Lb. helveticus, Lc. piscium, and Acinetobacter johnsonii; and cluster 3 had more $L c$. lactis, Lc. piscium, Lb. helveticus, and Lc. raffinolactis. The results revealed 4 highly variable tastes (sweetness, sourness, saltiness, and bitterness) among the studied samples, suggesting that the taste of jiaoke is complex and diverse. The taste quality might be associated with its microbial community (particularly LAB) because of microbial metabolism. For example, the composition of cheese microbiota might influence amino acid and fat metabolism in cheese and thus its taste (McSweeney, 2004; Piombino et al., 2008). Cluster 2 was distinct from clusters 1 and 3, as shown on the weighted UniFrac PCoA score plot. The LAB microbiota of the 3 taste clusters was thus analyzed. No significant difference was noted among the 3 groups at the phylum level. However, differences were apparent at the species level. Cluster 2 had significantly more Lc. lactis and $L b$. helveticus. Interesting correlations were found between sequences representing specific jiaoke microbes and taste quality. In particular, 4 of the monitored taste qualities showed great variation among samples, including bitterness, saltiness, sourness, and sweetness.

We found interesting correlations between some LAB and taste qualities. For example, Lc. piscium correlated positively with umami, while correlated negatively with aftertaste-B and aftertaste-A. Lactobacillus helveticus correlated negatively with bitterness, saltiness, and umami, whereas Lc. lactis correlated positively with aftertaste-B. Fall et al. (2012) did not detect histamine and tyramine in fermentation products containing $L c$. piscium. Histidine, valine, leucine, and phenylalanine are bitter amino acids, and histamine and tyramine are the main bitter biogenic amines (Suzuki et al., 2004).
These studies indirectly confirmed that $L c$. piscium correlated negatively with aftertaste-B (bitterness of aftertaste). The relative abundance of $L b$. helveticus correlated negatively with bitterness. Bitterness is caused by an imbalance in proteolysis and peptide hydrolysis in dairy products such as cheese (Zhao et al., 2016), especially relating to the proteolysis of $\beta$-casein, which results in accumulation of bitter peptides, leading to the bitter taste (FitzGerald and O'Cuinn, 2006). Debittering in food fermentation is achieved by introducing or strengthening peptidase activities to lower the level of bitter peptides (Sridhar et al., 2005). Lactobacillus helveticus can exhibit debittering effect via its prolyl endopeptidases, such as PepO2, PepO3, and PepF (Chen et al., 2003). Although jiaoke and cheese are made in different ways, the negative correlation between $L b$. helveticus and bitterness might indicate a similar role for this species in influencing the taste of jiaoke. In contrast, Lc. lactis was correlated positively with bitterness probably because of its extracellular proteases, which have been shown to enhance accumulation of bitter peptides under certain conditions (Broadbent et al., 2002).

Saltiness and sourness may affect the perception of bitterness (Zhao et al., 2016). Sequences representing LAB comprised up to $83.73 \%$ of the total sequences of jiaoke microbiota. A high proportion of $\mathrm{LAB}$ release significant amounts of lactic acid, which leads to high acidity and possibly bitterness of fermented products. This acidic ecological environment does not favor the survival of most harmful microorganisms, which helps maintain the long shelf life of jiaoke (Chen et al., 2019). Despite this, a small proportion of the detected sequences represented potentially harmful bacteria; for example, Macrococcus caseolyticus and K. pneumoniae, which could have originated from the open production environment. The viability of these potential contaminants was not verified in this work. Nevertheless, it is important to optimize the processing and hygiene environment of jiaoke production. This study provides a comprehensive description of jiaoke microbiota and its link to the taste of the product.

The current work had several limitations. For example, because there were practical difficulties in sampling jiaoke, it was done based on household availability. Thus, the current results represent only the jiaoke microbiota and LAB population present in samples collected at visited locations at a specific time point. Further sampling is necessary to gain a more comprehensive overview of the microbial community that exists in this type of product. Moreover, because our methodology did not incorporate any tests to trace the origin of the detected microbial communities, they could have come from the raw material of jiaoke, the 
manufacturing utensils, the direct environment, or even the person who made the jiaoke.

\section{CONCLUSIONS}

This study combined SMRT sequencing and traditional bacterial culture methods to describe the microbiota of jiaoke collected from Inner Mongolia. A rich microbial diversity was found in jiaoke, particularly $\mathrm{LAB}$, demonstrating that it is a good source for isolating novel LAB strains. By using an electronic tongue device, 4 taste qualities-bitterness, sourness, saltiness, and sweetness - were shown to vary greatly among the collected samples. Finally, interesting correlations were identified between some microbial taxa and specific taste qualities, indicating the important role of jiaoke microbes in shaping the product's taste.

\section{ACKNOWLEDGMENTS}

This research was supported by the Earmarked fund for the National Key R \& D Program of 13th Five-Year Plan of China (Grant No. 2018YFD0400102), the National Natural Science Foundation of China (Grant No. 31571814), and the China Agriculture Research System (Grant CARS-36). The authors have not stated any conflicts of interest.

\section{REFERENCES}

Adelowo, O. O., and O. Fagade. 2009. The tetracycline resistance gene tet39 is present in both Gram-negative and Gram-positive bacteria from a polluted river, Southwestern Nigeria. Lett. Appl. Microbiol. 48:167-172. https://doi.org/10.1111/j.1472-765X.2008.02523.x.

Aldrete-Tapia, A., M. C. Escobar-Ramírez, M. L. Tamplin, and M. Hernández-Iturriaga. 2014. High-throughput sequencing of microbial communities in Poro cheese, an artisanal Mexican cheese. Food Microbiol. 44:136-141. https://doi.org/10.1016/j.fm.2014.05 .022 .

Broadbent, J. R., M. Barnes, C. Brennand, M. Strickland, K. Houck, M. E. Johnson, and J. L. Steele. 2002. Contribution of Lactococcus lactis cell envelope proteinase specificity to peptide accumulation and bitterness in reduced-fat Cheddar cheese. Appl. Environ. Microbiol. 68:1778-1785. https://doi.org/10.1128/AEM.68.4.1778 $-1785.2002$.

Caliński, T., and J. Harabasz. 1974. A dendrite method for cluster analysis. Commun. Stat. Theory Methods 3:1-27. https://doi.org/ 10.1080/03610927408827101.

Caporaso, J. G., K. Bittinger, F. D. Bushman, T. Z. DeSantis, G. L. Andersen, and R. Knight. 2010a. PyNAST: A flexible tool for aligning sequences to a template alignment. Bioinformatics 26:266-267. https://doi.org/10.1093/bioinformatics/btp636.

Caporaso, J. G., J. Kuczynski, J. Stombaugh, K. Bittinger, F. D. Bushman, E. K. Costello, N. Fierer, A. G. Pena, J. K. Goodrich, J. I. Gordon, G. A. Huttley, S. T. Kelley, D. Knights, J. E. Koenig, R. E. Ley, C. A. Lozupone, D. McDonald, B. D. Muegge, M. Pirrung, J. Reeder, J. R. Sevinsky, P. J. Turnbaugh, W. A. Walters, J. Widmann, T. Yatsunenko, J. Zaneveld, and R. Knight. 2010b. QIIME allows analysis of high-throughput community sequencing data. Nat. Methods 7:335-336. https://doi.org/10.1038/nmeth.f .303 .
Casalta, E., J.-M. Sorba, M. Aigle, and J.-C. Ogier. 2009. Diversity and dynamics of the microbial community during the manufacture of Calenzana, an artisanal Corsican cheese. Int. J. Food Microbiol. 133:243-251. https://doi.org/10.1016/j.ijfoodmicro.2009.05.022.

Cavanagh, D., G. F. Fitzgerald, and O. McAuliffe. 2015. From field to fermentation: the origins of Lactococcus lactis and its domestication to the dairy environment. Food Microbiol. 47:45-61. https:// doi.org/10.1016/j.fm.2014.11.001.

Chen, C., Y. Lu, H. Yu, Z. Chen, and H. Tian. 2019. Influence of 4 lactic acid bacteria on the flavor profile of fermented apple juice. Food Biosci. 27:30-36. https://doi.org/10.1016/j.fbio.2018.11.006.

Chen, Y.-S., J. E. Christensen, J. R. Broadbent, and J. L. Steele. 2003. Identification and characterization of Lactobacillus helveticus $\mathrm{PepO}$, an endopeptidase with post-proline specificity. Appl. Environ. Microbiol. 69:1276-1282. https://doi.org/10.1128/AEM 69.2.1276-1282.2003.

Cole, J. R., B. Chai, R. J. Farris, Q. Wang, A. S. Kulam-Syed-Mohideen, D. M. McGarrell, A. M. Bandela, E. Cardenas, G. M. Garrity, and J. M. Tiedje. 2007. The ribosomal database project (RDP-II): Introducing myRDP space and quality controlled public data. Nucleic Acids Res. 35(suppl_1):D169-D172. https://doi.org/ $10.1093 /$ nar/gkl889.

Dan, T., H. Chen, T. Li, J. Tian, W. Ren, H. Zhang, and T. Sun. 2019. Influence of Lactobacillus plantarum P-8 on fermented milk flavor and storage stability. Front. Microbiol. 9:3133.

del Rio, B., V. Ladero, B. Redruello, D. M. Linares, M. Fernández, M. C. Martín, and M. A. Alvarez. 2015. Lactose-mediated carbon catabolite repression of putrescine production in dairy Lactococcus lactis is strain dependent. Food Microbiol. 48:163-170. https://doi .org/10.1016/j.fm.2014.11.018.

DeSantis, T. Z., P. Hugenholtz, N. Larsen, M. Rojas, E. L. Brodie, K. Keller, T. Huber, D. Dalevi, P. Hu, and G. L. Andersen. 2006. Greengenes, a chimera-checked 16S rRNA gene database and work-bench compatible with ARB. Appl. Environ. Microbiol. 72:5069-5072. https://doi.org/10.1128/AEM.03006-05.

Edgar, R. C. 2010. Search and clustering orders of magnitude faster than BLAST. Bioinformatics 26:2460-2461. https://doi.org/10 $.1093 /$ bioinformatics/btq461.

Fall, P. A., M. F. Pilet, F. Leduc, M. Cardinal, G. Duflos, C. Guérin, J.-J. Joffraud, and F. Leroi. 2012. Sensory and physicochemical evolution of tropical cooked peeled shrimp inoculated by Brochothrix thermosphacta and Lactococcus piscium CNCM I-4031 during storage at 8 C. Int. J. Food Microbiol. 152:82-90. https://doi.org/ 10.1016/j.ijfoodmicro.2011.07.015.

FitzGerald, R., and G. O'Cuinn. 2006. Enzymatic debittering of food protein hydrolysates. Biotechnol. Adv. 24:234-237. https://doi .org/10.1016/j.biotechadv.2005.11.002.

Gesudu, Q., Y. Zheng, X. Xi, Q. C. Hou, H. Xu, W. Huang, H. Zhang, B. Menghe, and W. Liu. 2016. Investigating bacterial population structure and dynamics in traditional koumiss from Inner Mongolia using single molecule real-time sequencing. J. Dairy Sci. 99:7852-7863. https://doi.org/10.3168/jds.2016-11167.

Gong, H., X. Meng, and H. Wang. 2010. Plantaricin MG active against Gram-negative bacteria produced by Lactobacillus plantarum KLDS1. 0391 isolated from "Jiaoke", a traditional fermented cream from China. Food Control 21:89-96. https://doi.org/10.1016/j foodcont.2009.04.005.

Gupta, M., and B. K. Bajaj. 2017. Development of fermented oat flour beverage as a potential probiotic vehicle. Food Biosci. 20:104-109. https://doi.org/10.1016/j.fbio.2017.08.007.

Hou, Q., C. Li, Y. Liu, W. Li, Y. Chen, Y. Bao, H. Zhang, B. Menghe, and Z. Sun. 2019. Koumiss consumption modulates gut microbiota, increases plasma high density cholesterol, decreases immunoglobulin G and albumin. J. Funct. Foods 52:469-478. https:// doi.org/10.1016/j.jff.2018.11.023.

Hou, Q., H. Xu, Y. Zheng, X. Xi, L.-Y. Kwok, Z. Sun, H. Zhang, and W. Zhang. 2015. Evaluation of bacterial contamination in raw milk, ultra-high temperature milk and infant formula using single molecule, real-time sequencing technology. J. Dairy Sci. 98:84648472. https://doi.org/10.3168/jds.2015-9886. 
Hui, W., Q. Hou, C. Cao, H. Xu, Y. Zhen, L. Y. Kwok, T. Sun, H. Zhang, and W. J. J. Zhang. 2017. Identification of microbial profile of koji using single molecule, real-time sequencing technology. J. Food Sci. 82:1193-1199. https://doi.org/10.1111/1750-3841.13699.

Jin, H., L. Mo, L. Pan, Q. Hou, C. Li, I. Darima, and J. Yu. 2018. Using PacBiol. sequencing to investigate the bacterial microbiota of traditional Buryatian cottage cheese and comparison with Italian and Kazakhstan artisanal cheeses. J. Dairy Sci. 101:6885-6896. https://doi.org/10.3168/jds.2018-14403.

Kumar, S., G. Stecher, and K. Tamura. 2016. MEGA7: Molecular Evolutionary Genetics Analysis Version 7.0 for Bigger Datasets. Mol. Biol. Evol. 33:1870-1874. https://doi.org/10.1093/molbev/ msw054.

Le Boucher, C., F. Courant, S. Jeanson, S. Chereau, M.-B. Maillard, A.-L. Royer, A. Thierry, G. Dervilly-Pinel, B. Le Bizec, and S. Lortal. 2013. First mass spectrometry metabolic fingerprinting of bacterial metabolism in a model cheese. Food Chem. 141:10321040. https://doi.org/10.1016/j.foodchem.2013.03.094.

Li, J., Y. Zheng, H. Xu, X. Xi, Q. Hou, S. Feng, L. Wuri, Y. Bian, Z. Yu, L.-Y. Kwok, Z. Sun, and T. Sun. 2017. Bacterial microbiota of Kazakhstan cheese revealed by single molecule real time (SMRT) sequencing and its comparison with Belgian, Kalmykian and Italian artisanal cheeses. BMC Microbiol. 17:13. https://doi.org/10 $.1186 / \mathrm{s} 12866-016-0911-4$

Liu, W., Q. Bao, M. Qing, X. Chen, T. Sun, M. Li, J. Zhang, J. Yu, M. Bilige, and T. Sun. 2012. Isolation and identification of lactic acid bacteria from Tarag in Eastern Inner Mongolia of China by 16S rRNA sequences and DGGE analysis. Microbiol. Res. 167:110-115. https://doi.org/10.1016/j.micres.2011.05.001.

Liu, W., Y. Zheng, L.-Y. Kwok, Z. Sun, J. Zhang, Z. Guo, Q. Hou, B. Menhe, and H. Zhang. 2015. High-throughput sequencing for the detection of the bacterial and fungal diversity in Mongolian naturally fermented cow's milk in Russia. BMC Microbiol. 15:45. https://doi.org/10.1186/s12866-015-0385-9.

Lozupone, C., and R. J. A. E. M. Knight. 2005. UniFrac: A new phylogenetic method for comparing microbial communities. Appl. Environ. Microbiol. 71:8228-8235. https://doi.org/10.1128/AEM $.71 .12 .8228-8235.2005$.

Ma, J., J. Kong, and M. Ji. 2009. Detection of the lactic acid bacteria in commercial yoghurts by PCR-denaturing gradient gel electrophoresis. Chin. J. Appl. Environ. Biol. 15:534-539.

McSweeney, P. L. 2004. Biochemistry of cheese ripening. Int. J. Dairy Technol. 57:127-144. https://doi.org/10.1111/j.1471-0307.2004 .00147.x.

Milani, E., F. Shahidi, S. A. Mortazavi, and M. Saeedi. 2017. Isolation and identification of lactic acid bacteria in Kurdish cheese during ripening using $16 \mathrm{~S}$ rRNA gene sequence analysis. J. Food Process. Preserv. 41:e13009. https://doi.org/10.1111/jfpp.13009.

Mosher, J. J., E. L. Bernberg, O. Shevchenko, J. Kan, and L. A. Kaplan. 2013. Efficacy of a 3rd generation high-throughput sequencing platform for analyses of $16 \mathrm{~S}$ rRNA genes from environmental samples. J. Microbiol. Methods 95:175-181. https://doi.org/10 .1016/j.mimet.2013.08.009.

Nam, Y. D., S. Park, and S. I. Lim. 2012. Microbial composition of the Korean traditional food "kochujang" analyzed by a massive sequencing technique. J. Food Sci. 77:M250-M256. https://doi.org/ 10.1111/j.1750-3841.2012.02656.x.

National Standards of the People's Republic of China. 1996. Method for analysis of hygienic standard of milk and milk products. Bulletin 5009:46; China National Standards, Beijing, China.

Piombino, P., R. Pessina, A. Genovese, M. Lisanti, and L. Moio. 2008. Sensory profiling, volatiles and odor-active compounds of Canestrato Pugliese PDO cheese made from raw and pasteurized ewes' milk. Ital. J. Food Sci. 20:225-237.
Price, M. N., P. S. Dehal, and A. P. Arkin. 2009. FastTree: Computing large minimum evolution trees with profiles instead of a distance matrix. Mol. Biol. Evol. 26:1641-1650. https://doi.org/10.1093/ molbev/msp077.

Quigley, L., O. O'Sullivan, T. P. Beresford, R. P. Ross, G. F. Fitzgerald, and P. D. Cotter. 2012. High-throughput sequencing for detection of subpopulations of bacteria not previously associated with artisanal cheeses. Appl. Environ. Microbiol. 78:5717-5723. https:/ /doi.org/10.1128/AEM.00918-12.

Quirós, C., M. Herrero, L. A. García, and M. Díaz. 2009. Quantitative approach to determining the contribution of viable-but-nonculturable subpopulations to malolactic fermentation processes. Appl. Environ. Microbiol. 75:2977-2981. https://doi.org/10.1128/AEM .01707-08.

Rein, M. 2018. The perceived taste of lactic acid fermentation in protein hydrolysates: a screening of the sensory properties of lactic acid bacteria (LAB) implemented in hydrolysate products produced with co-products from the food industry (poultry, fish, and dairy). MS Thesis. Norwegian University of Life Sciences, Ås, Norway.

Schornsteiner, E., E. Mann, O. Bereuter, M. Wagner, and S. SchmitzEsser. 2014. Cultivation-independent analysis of microbial communities on Austrian raw milk hard cheese rinds. Int. J. Food Microbiol. 180:88-97. https://doi.org/10.1016/j.ijfoodmicro.2014 .04 .010 .

Sridhar, V., J. Hughes, D. Welker, J. Broadbent, and J. Steele. 2005. Identification of endopeptidase genes from the genomic sequence of Lactobacillus helveticus CNRZ32 and the role of these genes in hydrolysis of model bitter peptides. Appl. Environ. Microbiol. 71:3025-3032. https://doi.org/10.1128/AEM.71.6.3025-3032.2005.

Sun, Z., W. Liu, Q. Bao, J. Zhang, Q. Hou, L. Kwok, T. Sun, and H. Zhang. 2014. Investigation of bacterial and fungal diversity in tarag using high-throughput sequencing. J. Dairy Sci. 97:60856096. https://doi.org/10.3168/jds.2014-8360.

Suzuki, H., K. Kato, and H. Kumagai. 2004. Enzymatic synthesis of $\gamma$-glutamylvaline to improve the bitter taste of valine. J. Agric. Food Chem. 52:577-580. https://doi.org/10.1021/jf0347564.

Wang, Y., K. Dai, X. Shen, Y. Dong, S. Zhou, and Z. Guo. 2018. Characterization of fungal microflora and its influence on taste quality of zhaguangjiao, a Chinese traditional fermented chili product. Shipin Kexue/Food Sci. 39:173-178.

Watanabe, K., J. Fujimoto, M. Sasamoto, J. Dugersuren, T. Tumursuh, and S. Demberel. 2008. Diversity of lactic acid bacteria and yeasts in Airag and Tarag, traditional fermented milk products of Mongolia. World J. Microbiol. Biotechnol. 24:1313-1325. https:// doi.org/10.1007/s11274-007-9604-3.

Wickham, H. 2016. ggplot2: Elegant graphics for data analysis. Springer Nature, Berlin, Germany.

Wu, R., L. Wang, J. Wang, H. Li, B. Menghe, J. Wu, M. Guo, and H. Zhang. 2009. Isolation and preliminary probiotic selection of lactobacilli from koumiss in Inner Mongolia. J. Basic Microbiol. 49:318-326. https://doi.org/10.1002/jobm.200800047.

Yamei, Y.-S. Guo, J.-J. Zhu, F. Xiao, J.-P. Sun, J.-P. Qian, W.-L. $\mathrm{Xu}$, C.-D. Li, and L. Guo. 2019. Investigation of physicochemical composition and microbial communities in traditionally fermented vrum from Inner Mongolia. J. Dairy Sci. 102:8745-8755. https:// doi.org/10.3168/jds.2019-16288. PubMed

Zhao, C. J., A. Schieber, and M. G. Gänzle. 2016. Formation of tasteactive amino acids, amino acid derivatives and peptides in food fermentations - A review. Food Res. Int. 89:39-47. https://doi .org/10.1016/j.foodres.2016.08.042.

Zhu, J., F. Xiao, and M. Ya. 2017. Inner Mongolia traditional milk fat process and nutrition analysis. Farm Prod. Process. 3:63-65. 